\title{
Pluralist Structural Realism: The Best of Both Worlds?
}

\section{David Glick}

Received: 13 June 2018 / Accepted: 17 July 2019

\begin{abstract}
John Worrall (1989) famously claimed that structural realism is the best of both worlds; it enables one to endorse the best arguments for scientific realism and antirealism. In this paper, I argue that structural realism also enables one to combine two other seemingly inconsistent positions: realism and pluralism. Indeed, the very features which form the basis of the structural realist's reply to the problem of theory change may be applied synchronically to allow for a pluralist structural realism. The resulting position incorporates a robust variety of scientific pluralism unavailable to the traditional realist and thereby allows the structural realist to take seriously the pluralism apparent in scientific practice.
\end{abstract}

\section{Introduction}

A flat-footed look at scientific practice reveals a kind of pluralism; it sometimes happens that multiple models or theories are applied to the same target. For example, Morrison (2011) describes the plurality of models of turbulent flows used in fluid dynamics and the diversity of incompatible models for the atomic nucleus deployed by physicists. ${ }^{1}$ Philosophers of science may take a variety of attitudes toward such pluralism in practice, but for those who wish to take

This is a post-peer-review, pre-copyedit version of an article published in Synthese. The final authenticated version is available online at: https://doi.org/10.1007/s11229-019-02333-3

David Glick

Department of Philosophy

University of Sydney

E-mail: david.glick@sydney.edu.au

1 Morrison notes that there is an important difference between these two cases - the former models are compatible in a way the latter aren't - but at this stage, the point is simply that both serve as examples of pluralism in practice. A number of examples from biology are given by Mitchell (2003) in defense of her 'integrative pluralism'. See also Kellert et al. (2006) for examples from across the sciences. 
current scientific practice as a guide, there is some motivation to incorporate pluralism in one's philosophy of science - that is, to regard pluralism as a feature of healthy science rather than a defect or temporary phase in scientific development.

One challenge in endorsing pluralism is the apparent conflict with scientific realism. Below I will argue that the conflict is only apparent. If one adopts a form of structural realism, pluralism can be reconciled with realism. Moreover, the variety of pluralism allowed by structural realism - 'robust scientific pluralism' - is stronger than what the traditional realist can admit. This is a benefit insofar as one seeks to take seriously the pluralism found in our scientific practice. Thus, pluralism provides another path to structural realism apart from the historical problem of theory change and issues in contemporary physics often appealed to by structural realists.

The paper will proceed as follows. The remainder of this section aims to clarify the challenge to scientific realism presented by pluralism. Section 2 characterizes robust scientific pluralism and distinguishes it from other forms of scientific pluralism. Section 3 briefly outlines structural realism, which I understand as a stance in the sense of van Fraassen's (2008a) empirical stance. In section 4 , I argue that structural realism - both in several extant accounts and more generally - can allow for robust scientific pluralism. The resulting position, pluralist structural realism, does not require any substantial modification of structural realism, but rather follows from the structural realist's antecedent commitments and aims. I then consider two further questions facing pluralist structural realism: (a) what makes a certain plurality of theories or models acceptable? (section 5) and (b) is pluralist structural realism still realism? (section 6). Finally, I conclude with a brief application to a contemporary scientific theory that has challenged realist interpreters: algebraic quantum field theory (section 7).

\subsection{The pluralist challenge}

To demonstrate the conflict between pluralism and realism, consider the following rough characterizations:

Pluralism: One can accept multiple inequivalent theories dealing with the same phenomenon.

Realism: Acceptance of a theory involves a commitment to it providing a faithful representation of the world.

Immediately one can see a tension between pluralism and realism. Pluralism allows for the acceptance of a plurality of theories that (a) have the same target and (b) are genuinely inequivalent. The former condition rules out a sort of piecemeal approach in which different theories deal with different bits of the world and hence never come in conflict (cf., Cartwright's (1999) 'dappled world'). The latter rules out the possibility that the plurality may be reduced to a single theoretical account of the phenomena that admits of 
several presentations. The content of realism turns on what counts as a 'faithful representation' of the world. If one takes it to be strict literal truth (in the correspondence sense), then combining realism with pluralism seems to require giving up either (a) classical logic and/or (b) metaphysical realism. Suppose theories in the plurality disagree about some proposition $p$. Then, if we take them all to be strictly true, $p \wedge \neg p$ will be true. Another apparent consequence would be that the world is such that $p$ and such that $\neg p$, contra the assumption of metaphysical realism, i.e., that there is mind-independent world free of contradictory states of affairs. ${ }^{2}$

Of course, scientific realists typically regard approximate truth as the relevant theory/world relation, and it's less clear that this precludes pluralism. One reason for this is a lack of consensus in the realist's notion of approximate truth. But, regardless of how the details are spelled out, the appeal to approximate truth doesn't obviously avoid the tension with pluralism. For instance, one could follow Larry Laudan in claiming that "a realist would never want to say that a theory was approximately true if its central theoretical terms failed to refer" (Laudan, 1981, 33). More generally, it's difficult to make sense of how theories with different core ontological commitments can each be taken to approximate the world on the traditional realist picture. Intuitively, such theories assert that there are different things in the world, so it doesn't seem possible that more than one can capture what the world is like, even approximately. Indeed, the notion of one ontology approximating another seems altogether mysterious. ${ }^{3}$

The forgoing falls short of establishing the incompatibility of pluralism and realism, even when the latter is understood in terms of approximate truth. As we will see in the next section, there are moves open to the realist who seeks to incorporate some degree of pluralism. The point is rather that the realist faces an apparent challenge: how can one coherently accept both pluralism and realism without compromising either thesis? Below, I will argue that structural realism allows one to answer the challenge in a way unavailable to the traditional realist. Before doing so, however, let's consider how the traditional realist may respond to the challenge. This will also serve to clarify what is required of a robust scientific pluralism.

\footnotetext{
2 The issue of inconsistency in science is complex and deserving of a careful analysis. Here I simply take it for granted that inconsistent collections of theories or models are used in science and that we wish to preserve classical logic. For a detailed investigation of inconsistency in science, see Vickers (2013).

3 I return to this point in section 4.3 below. For now, note that the traditional scientific realist endorses the unobservable ontology of accepted theories - indeed, this is sometimes taken as analytic - which precludes acceptance of ontologically-incompatible theories. Thus, the traditional realist must either abandon their view of ontological commitment or limit the pluralism they are willing to admit.
} 


\section{Robust scientific pluralism}

Two key concepts that figure in the initial statements of pluralism and realism above deserve further scrutiny: acceptance and equivalence. In this section, I will argue that the traditional realist can understand each of these terms in a way that will permit a form of pluralism, but in doing so threatens to compromise one or both of the theses.

\subsection{Acceptance}

If acceptance requires something less than truth, then a plurality of inequivalent theories can be accepted without fear of contradiction. However, as a general position, this move threatens to conflict with realism. It is central to the traditional realist understanding that the acceptance of a scientific theory involves the belief that it is true, at least approximately. Perhaps, then, the realist should restrict their claim to the context in which the plurality of theories occurs. On such a view one cannot accept a plurality of inequivalent theories, but can nevertheless 'take them on' or 'entertain' them in some sense. Thus, we arrive at a hybrid approach. Ordinarily, acceptance of theories involves a commitment to their truth, but in permissible cases of pluralism acceptance lacks such commitment. While such a view may be able to make sense of the pluralism of practice, it does so at the cost of robust pluralism by denying the following condition.

Parity: One should be willing to accept multiple inequivalent theories in the same sense of 'acceptance' as appropriate to theories taken in isolation.

Note that Parity does not require the presence of other theories to be irrelevant; there are certainly situations in which the status we give to a theory should be affected by the presence of rivals. ${ }^{4}$ The point is rather that a robust pluralist must be willing to treat more than one theory as adequate in the same sense as a single theory is adequate. For the traditional realist, such adequacy involves approximate truth, so Parity requires that inequivalent theories can each be approximately true, in contrast to the hybrid strategy under consideration.

\subsection{Equivalence}

Another strategy open to the realist is to scrutinize the sense in which the theories endorsed by the pluralist are inequivalent. One instance of this strategy seeks to render inequivalent theories consistent via a reinterpretation. If superficially inequivalent theories agree at a deeper level, then the traditional realist is free to endorse all of the them. There are several problems with this approach, however. First, reinterpretation of this sort threatens to be ad hoc

\footnotetext{
4 Although, it's often difficult to say when. See section 5 below.
} 
and capable of rendering any theories equivalent when 'properly understood'. Second, a central feature of scientific realism is its willingness to take theories at face-value and commit to their literal (approximate) truth. A willingness to reinterpret theories to resolve conflicts is a strategy more associated with forms of antirealism. Third, even if one allows for such a reinterpretation, the resulting pluralism is quite weak. Given that the theories agree once interpreted properly, the sense in which they are inequivalent is to a certain extent only apparent; ultimately, there is only one representation of the world that admits of more than one formulation. This runs afoul of another condition on robust pluralism:

Content: One should allow for theories which are genuinely inequivalent by the lights of the version of realism or antirealism one adopts.

Content rules out versions of pluralism — such as that just sketched - which allow for theories that are inequivalent by the lights of others, but equivalent by the lights of one's own view. This captures the sense in which pluralism should be a more substantive thesis than merely admitting of multiple formulations or notational variants of a single theory. For example, a logical positivist may readily accept several empirically equivalent theories regarded as inequivalent by the realist, but in so doing does not endorse a robust form of pluralism. By contrast, a constructive empiricist (in the sense of van Fraassen (1980)) may regard empirically equivalent theories as genuinely inequivalent, provided they differ in what they say about the unobservable. ${ }^{5}$ Thus, for the constructive empiricist, Content would be satisfied by allowing for the acceptance of such a plurality, which includes theories that are inequivalent by their own lights. ${ }^{6}$

Content does not rule out all equivalence-based strategies for meeting the challenge of pluralism. For example, Chakravartty (2011), discusses a metaphysical-natures based pluralism according to which theories may disagree about the details of their shared (core) ontological commitments. Consider two theories that both mention electrons, but ascribe different features to them. Even if we grant that wholesale ontological inequivalence is problematic (for the reasons given above), it seems that these theories can each be approximately true, as long as (a) there are electrons and (b) their features are approximately like those described by both theories under consideration. Thus, there does seem to be room for a version of pluralism that allows for theories that are inequivalent with respect to metaphysical-natures but equivalent in their core ontological commitments.

\footnotetext{
5 And, because the constructive empiricist is committed to semantic realism, they will agree with the (traditional) realist about what theories say about the (putative) unobservable.

6 This would require a departure from van Fraassen's original version of the view, which holds that certain non-doxastic commitments preclude simultaneously accepting more than one theory in a given area of inquiry (van Fraassen, 1980, 88). Note as well that matters are different on the empiricist structuralism of van Fraassen (2008b). On this view, theories are understood in terms of abstract structures describable only up to isomorphism (238). Thus, allowing for a plurality of empirically equivalent theories that differ in their traditional realist content may not satisfy Content; one would have to add that the members of the plurality are non-isomorphic.
} 
Metaphysical-natures pluralism must walk a fine line between allowing genuine inequivalence and avoiding disagreement about core ontology. Consider again the case of electrons. Chakravartty regards a theory that takes subatomic particles to be field excitations and another that takes them to be roughly similar to classical particles as disagreeing not about what there is, but about its metaphysical nature. However, even if one isn't an essentialist about electrons, this understanding is somewhat strained. Rival understandings of quantum field theory do seem to disagree about fundamental ontology; defenders of a field interpretation think that fundamentally there are no particles and that they - or particle-like phenomena - only emerge at a suitable level of description. $^{7}$ Moreover, the metaphysical-natures view also faces a difficult choice between underdetermination and indeterminacy about fundamental ontology. On this view, electrons either (a) have metaphysical natures about which we cannot know or (b) have genuinely indeterminate metaphysical natures. The former option introduces an unwelcome element of humility into realism; our best scientific theories are incapable of providing a full account of how things are. The latter option eschews this humility, but does so at the cost of positing metaphysical indeterminacy, a quite controversial commitment.

\subsection{Robust scientific pluralism}

A robust scientific pluralism (RSP) should respect both Parity and Content. It must be open to the pluralist to accept - in the relevant sense of 'acceptance' a plurality of genuinely inequivalent theories in a given area. It's worth noting that robust scientific pluralism differs from other notions of scientific pluralism that have been discussed in the literature. First, consider methodological pluralism. According to methodological pluralism, one should allow-or perhaps promote - the pursuance of a plurality of theories in a given area of inquiry. Methodological pluralism is a significantly weaker thesis than robust scientific pluralism. It is compatible with-perhaps even recommended by - an underlying monistic view according to which there is one best theory to be found; of course we should pursue multiple avenues until we hit upon the correct one! ${ }^{8}$ $\mathrm{RSP}$ is stronger because it allows not only pursuance but acceptance of the plurality. By rejecting Parity, methodological pluralism fails to be robust.

Second, consider what is sometimes called metaphysical pluralism. This view rejects metaphysical monism, the thesis that there is one way the world is (fundamentally). ${ }^{9}$ Metaphysical pluralism isn't about our theories or models, but rather the world itself; it claims that (somehow) our world is both $P$ and $Q$, where these are genuinely inequivalent ways of being. Whether such a metaphysical pluralism is coherent is a matter of debate, but it's certainly

\footnotetext{
7 For arguments against particle and field interpretations, respectively, see Fraser (2008) and Baker (2009).

8 Cf., Chang (2012, 270-271), who defends this sort of reasoning in terms of bet hedging.

9 Perhaps the 'promiscuous realism' of Dupré (1993) is a version of metaphysical pluralism.
} 
stronger than RSP. Below I will argue that RSP is compatible with metaphysical monism. For now, note that RSP is a claim about our stance toward scientific theories. ${ }^{10}$ It holds that we can adopt the same stance toward a genuine plurality of theories that one adopts toward a successful theory taken in isolation. This might have implications for metaphysics, but these need to be carefully worked out. Metaphysical pluralism would certainly result if one added to RSP the claim that theories provide a complete and perfectly accurate representation of the world, but no plausible form of realism would assert such a tight connection between theories and the world. In lieu of such a connection, the metaphysical consequences are less obvious; they will depend on the precise details of the theory/world relation.

Thus, RSP is situated roughly between methodological and metaphysical pluralism. It deserves the title 'robust' because it requires the genuine acceptance of inequivalent theories, but it is not, in the first instance, a metaphysical thesis. Another version of pluralism that seeks this middle ground is the active normative epistemic pluralism of Chang (2012), which may be characterized roughly as follows.

I would define pluralism in science as the doctrine advocating the cultivation of multiple systems of practice in any given field of science. By a 'system of practice' I mean a coherent and interacting set of epistemic activities performed with a view to achieve certain aims. (Chang, 2012, 260)

Chang's view shares some important features with RSP. For instance, his pluralism does not presuppose metaphysical pluralism or that the world is highly complex (Chang, 2012, 292-293). This is consistent with RSP, which also avoids (direct) commitments about the nature of reality. However, there are at least two important contrasts with RSP. The first is that Chang's pluralism is more active and normative than required by RSP. Whereas he advocates for the use of a plurality of scientific systems, RSP claims only that pluralities of theories may, in certain circumstances, be acceptable in the same way a single theory is. As discussed below, RSP doesn't provide explicit necessary and sufficient conditions for when a plurality is acceptable, nor does it claim that pluralities are epistemically superior to single theories in general. Second, Chang's pluralism concerns the cultivation of systems of practice, which is quite different from the theory-based (or model-based) understanding of RSP. On RSP, the plurality comprises inequivalent theories or models that may be accepted, which is quite different from Chang's broadly pragmatist conception of cultivating systems of practice. Chang emphasizes that his pluralism does include an epistemic component, but this seems to fall short of acceptance, at least when the latter is understood in realist terms.

RSP aims to take to heart the practices that motivate scientific pluralism. The fact that scientists often work with multiple, inequivalent theoretical

\footnotetext{
10 By 'stance' I mean the attitude(s) one adopts toward a scientific theory. For instance, a traditional realist stance toward a theory $T$ would involve a commitment to the approximate truth of $T$, while an empiricist stance would not (van Fraassen, 2008a).
} 
accounts of a given range of phenomena is taken to show that multiple theories can be accepted, even when they are genuinely inequivalent. By contrast, methodological pluralism weakens acceptance as it applies to the plurality (thereby violating Parity). Metaphysical pluralism is compatible with RSP, but not entailed by it unless acceptance requires exact literal truth in the correspondence sense, which realists should reject. Chang's view doesn't neatly fall into either of these categories, but it clearly differs from RSP in important ways. RSP is compatible with various forms of empiricism or antirealism. However, while it's not especially difficult to see how robust pluralism might be embraced by the antirealist, ${ }^{11}$ the real challenge concerns whether some version of scientific realism is compatible with RSP. Can we accept theories that are inequivalent on a realist understanding of both key terms?

\section{Structural realism}

In the next section, I will argue that structural realism can accommodate RSP. ${ }^{12}$ Before doing so, we should get clear on what is meant by the former thesis. There have been many discussions of structural realism and nearly as many ways of understanding the position. The present understanding is motivated by the problem of theory change in the (general) philosophy of science.

The problem of theory change begins from the observation of discontinuity across large-scale theoretical changes in science. Clear examples of such discontinuity include the sort of revolutions that inspired Kuhn - the Copernican revolution, the chemical revolution, the special theory of relativity-but even more subtle cases seem to create a problem for the realist. One way to see the problem is by following Laudan (1981), for whom the history of science is replete with counterexamples to the realist inference from empirical success to approximate truth. If approximate truth requires successful reference (as noted above), and currently accepted theories faithfully represent what there is, then many past theories cannot be approximately true despite their empirical success. The standard example discussed by structural realists is drawn from the

\footnotetext{
11 If acceptance requires something less than (approximate) correspondence truth, there is no substantial difficulty in accepting a plurality of theories. Indeed, if acceptance is made sufficiently weak, antirealist pluralism collapses into methodological pluralism-i.e., the claim that we can use a plurality of theories or models of the same system.

12 To be clear, I will not argue here that structural realism is the correct account of scientific theories, or even that it withstands criticisms that allege it is untenable or collapses into traditional forms of realism or antirealism. There have been several such objections made to the viability of structural realism. Two well known objections are (1) that the distinction between 'structure' and 'nature' cannot be sustained (Psillos, 2006) and (2) Newman's objection that claims about the structure of reality are trivially true (Newman, 1928; Demopoulos and Friedman, 1985). Addressing these important objections would take us too far afield and distract from the central aim of the paper: to establish the compatibility of pluralism and structural realism.
} 
history of optics. ${ }^{13}$ Fresnel's theory of light, the story goes, views light as a disturbance in a mechanical ether and from features of this ether derives empirically successful equations of reflection and refraction. Today, we no longer believe such an ether exists, so we are apparently forced to regard Fresnel's theory as false - not even approximately true. Perhaps this pronouncement is not so troubling for the realist, but the real problem is that if such discontinuity is the norm in science, we would expect that we are currently in a situation not dissimilar from Fresnel-our best confirmed theories will also be superseded by ontologically inconsistent future theories. This is a version of the 'pessimistic meta-induction' that threatens to undermine the realist's claim that today's best confirmed scientific theories are approximately true.

The structural realist solution to the problem of theory change attempts to locate continuity across theory change by focusing on structure. The guiding intuition is nicely captured by Poincaré's remarks on Fresnel's equations:

They teach us now, as they did then, that there is such and such a relation between this thing and that; only, the something which we then called motion, we now call electric current. But these are merely names of the images we substituted for the real objects which Nature will hide for ever from our eyes. The true relations between these real objects are the only reality we can attain, and the sole condition is that the same relations shall exist between these objects as between the images we are forced to put in their place. If the relations are known to us, what does it matter if we think it convenient to replace one image by another? (Poincaré, 1905, 179)

Poincaré's idea was revived by Worrall to give rise to the contemporary structural realist position on Fresnel and Maxwell:

...although from the point of view of Maxwell's theory, Fresnel entirely misidentified the nature of light, his theory accurately described not just light's observable effects but its structure. There is no elastic solid ether. There is, however, from the later point of view, a (disembodied) electromagnetic field. The field in no clear sense approximates the ether, but disturbances in it do obey formally similar laws to those obeyed by elastic disturbances in a mechanical medium. Although Fresnel was quite wrong about what oscillates, he was, from this later point of view, right, not just about the optical phenomena, but right also that these phenomena depend on the oscillations of something or other at right angles to the light. (Worrall, 1989, 118, original emphasis)

The structural realist solution to the problem of theory change has been challenged from a number of directions and the case of Fresnel and Maxwell

\footnotetext{
13 A number of other episodes of theory change have been discussed by structural realists. For example: Ptolemaic and Copernican astronomy (Saunders, 1993), classical mechanics and special relativity (Brown 1993), classical and quantum electrodynamics (Lyre, 2004), phologiston theory and oxygen theory (Ladyman, 2011). In each of these cases, structural realists acknowledge that there is ontological discontinuity, but allege that some measure of continuity can be recovered at the level of structure.
} 
widely scrutinized. It is not my intention here to defend the claims of Poincaré and Worrall, but rather, to show how the version of structural realism they suggest allows for RSP. To that end, it will be useful to highlight two features of the putative solution to the problem of theory change offered by structural realism.

First, the solution involves making a concession to the antirealist. The structural realist grants that many of the ontological commitments of the traditional realist must be abandoned. The only aspect of a successful scientific theory toward which we should adopt a realist attitude is its structure, where that is understood as what is encoded by the equations of the theory. This is what allows the structural realist to shrug off the apparent ontological discontinuity as the result of the "images" with which we clothe the underlying structure. In particular, Laudan's worry is avoided because earlier theories describe something we still (and always will) believe in, namely, structure.

Second, the structural realist needn't claim that structure is preserved exactly. One may note that structures themselves can change during episodes of theory change (Redhead, 2001). Even in the case of Fresnel and Maxwell, one can only recover the equations of the former theory from the latter as a special case given certain assumptions (Saatsi, 2005). However, the structural realist only needs to claim that there is continuity to be found across changes in theory (Votsis, 2010). Just as the traditional realist only claims approximate truth for today's best confirmed theories, the structural realist should only claim that they approximately capture the structure of the world. Of course, making precise this notion of approximating the world's structure is a challenge for the structural realist, but the same could be said for the traditional realist's notion of approximate truth. Neither view is plausible if an exact match between the world and our best theories (or their models) is required.

\subsection{ESR and OSR}

The presentation so far has ignored the important distinction between epistemic and ontic varieties of structural realism (Ladyman, 1998). Roughly, epistemic structural realism (ESR) holds that structure is all we can know and ontic structural realism (OSR) that structure is all there $i s$. While the distinction between ESR and OSR is important for many purposes, it is orthogonal to our focus here; both forms of structural realism allow for RSP. The crucial point is that all structural realists, properly so-called, take a realist attitude toward structure and only structure. ${ }^{14}$ Whether they are theistic, agnostic or atheistic about putative non-structural features of the world is another matter. But, as I will argue below, it is this modest commitment, in conjunction with a claim about 'looseness of fit' between the theory and the world, that allows for pluralism.

\footnotetext{
14 As with pluralism, I understand structural realism as a stance. In particular, a stance that adopts a realist attitude toward structure and only structure.
} 
That my focus crosscuts this distinction is also made clear by considering the level at which RSP operates. Again, the pluralism I'm concerned to defend is not a metaphysical thesis; it concerns only the stance we take toward a plurality of theories. Thus, whether the world is 'just structure' or there is something supporting that structure is beyond its scope. In what follows I wish to remain neutral with respect to all such matters; the fundamental ontology of our world could comprise: (1) a single structure, (2) a plurality of structures, (3) a collection of objects and/or properties that realizes a single structure, (4) a collection of objects and/or properties that realizes a plurality of structures. At times I may tacitly assume one metaphysical picture or another for the sake of demonstration, but I hope to make it clear that nothing turns on it.

\section{Pluralist structural realism}

The structural realist is better positioned to respond to the challenge of pluralism than her traditional realist rival. In particular, RSP - as captured by Parity and Content - can be embraced by the structural realist.

Parity: One should be willing to accept multiple inequivalent theories in the same sense of 'acceptance' as appropriate to theories taken in isolation.

Suppose we have two inequivalent theories that both meet the (structural) realist's threshold for empirical success. Can the structural realist grant that both faithfully represent the world? The answer seems to be straightforwardly 'yes'. Consider the case of Heisenberg's matrix mechanics and Schrödinger's wave mechanics. According to Schrödinger's theory, the state of the systemrepresented by its wavefunction $\psi$-changes as a function of time and the operators that act on it are time-independent. By contrast, Heisenberg's matrix mechanics takes states to be constant and the operators to change as as a function of time. These two theories concern the same subject matter-the world of the very small described by quantum mechanics - and are inequivalent in that they disagree about the nature of states and operators. For the structural realist, however, there is no difficulty claiming that both describe the structure of the world. The reason why is that, as proven by Dirac and von Neumann, both represent the same structure: a Hilbert space acted on by linear operators. So, this represents one way in which the structural realist can adopt pluralism: two theories that are incompatible by the lights of the traditional realist are structurally equivalent (isomorphic). In such cases the structural realist has no difficulty saying both theories faithfully represent the structure of the world.

The pluralist may still want more, however. For one thing, those cases in which two theories are isomorphic may be relatively rare. In addition, it's not clear that the traditional realist has to regard them as distinct theories. In the above case, the realist may reasonably claim that matrix mechanics and wave mechanics are different descriptions of the same underlying theory (they are commonly referred to as the Schrödinger and Heisenberg pictures). 
More significantly, the pluralism involved in such cases is to a certain extent only apparent. Given that the structural realist cares only about structure, it's not clear what basis there could be for distinguishing between isomorphic theories. Just as the logical positivist identifies empirically equivalent theories, so it would seem the structural realist should identify isomorphic ones. ${ }^{15}$ This would seem to suggest that A and B aren't genuinely distinct. In such cases, there is one way the world is and one way (multiply described) the theory in question represents it as being.

Even if the structural realist is able to regard isomorphic theories as distinct, the variety of pluralism that results will be quite weak. In particular, it doesn't satisfy Content.

Content: One should allow for theories which are genuinely inequivalent by the lights of the version of realism or antirealism one adopts.

Only if a structural realist can allow for cases in which the plurality contains theories that are inequivalent by their own lights can they endorse RSP. Thus, it is not enough to point to cases of alleged isomorphic theories with different (traditional realist) ontological commitments - as some authors have - to establish robust pluralism. ${ }^{16}$ Can structural realism allow for such cases?

In considering this question, it's helpful to recall the discussion of theory change above. The structural realist solution to the problem of theory change claimed that earlier scientific theories could be rendered consistent with their successors. The key to restoring the continuity was to (a) jettison the commitment to an ontology of objects and intrinsic properties and (b) to find continuity at the level of structure. If this approach is successful in that context, one might wonder whether it can be adapted to make room for genuine structural realist pluralism. Indeed, it seems that if the structural realist's response to the problem of theory change is successful, then one already has an instance of RSP in which the relevant theories occur on either side of a significant theory change in a given area of interest. The optical theories of Fresnel and Maxwell, for instance, are non-isomorphic while each may be taken to faithfully represent the structure of the world. Of course, there is one significant difference between the cases: in the problem of theory change we sought diachronic consistency while pluralism concerns a synchronic plurality of theories.

Some aspects of the structural realist solution to theory change carry over straightforwardly to the synchronic case. For example, the traditional realist

\footnotetext{
15 Halvorson (2012), for instance, takes this to be a commitment of structural realism. He goes on to challenge isomorphism as a criterion of theoretical equivalence. However, one might imagine a structural realist who agrees with the traditional realist about theoretical equivalence, but differs in what they take acceptance to involve. Such a view would seek to combine semantic realism with a structuralist view of ontological commitment in the way van Fraassen (1980) sought to combine semantic realism with empiricism. On this variant of structural realism, the acceptance of isomorphic theories may be sufficient for RSP. I will ignore this possibility in what follows, but note here that it may provide another route to pluralist structural realism. Thanks to $\mathrm{xxxx}$ for this suggestion.

16 This is the sort of ontological pluralism discussed by McArthur (2006), for example.
} 
who wishes to allow for a plurality of theories faces the problem of competing ontologies. In the case of theory change, the problem is that getting the ontology right is a plausible necessary condition for approximate truth, but past theories don't meet this requirement (by the lights of currently accepted theories). Similarly, if current theories in a given field of inquiry disagree about ontology, at most one can be approximately true for the traditional realist. This worry is avoided by the structural realist. If only structure is taken to have representational import, then differences in (non-structural) ontology among rival theories pose no impediment to more than one faithfully representing the world. This allows for the kind of modest pluralism discussed aboveisomorphic theories with apparently different ontological commitments can be endorsed. But, how can theories that attribute different structures to the world both be endorsed?

To answer this question more needs to be said about the notion of a theory 'faithfully representing the world' according to structural realism. As with approximate truth for the traditional realist, there is no consensus among structural realists here. My strategy will be to consider two different approaches that have been discussed in the literature. I will argue that each allows for RSP. This leaves open the possibility of an alternative structural realist understanding of the theory/world relation without the features that enable RSP, but I will offer some reasons to think this is unlikely.

4.1 Partial structures, partial morphisms and quasi-truth

One account of the relations between scientific structures is the view developed and defended by Newton Da Costa and collaborators (da Costa and French, 2003; Bueno and da Costa, 2007). On this view, theoretical models are associated with partial structures that are related by partial morphisms and assessed in terms of quasi-truth. Such a view allows that inequivalent- even inconsistent - theories may each be quasi-true, allowing for a robust variety of pluralism.

In ordinary set theory, a structure is an ordered pair that consists of a domain $D$ and a set of relations $R_{i}$ that hold between them, that is, $S=$ $\left\langle D, R_{i}\right\rangle_{i \in I}$. Each of the relations $R_{i}$ is defined extensionally as a set of ( $n$ tuples of) the elements of $D$. Partial structures have the same form, except that relations are replaced by partial relations. An $n$-place partial relation $R$ is defined as a triple $\left\langle R_{1}, R_{2}, R_{3}\right\rangle$, where $R_{1}, R_{2}$, and $R_{3}$ are mutually disjoint sets, with $R_{1} \cup R_{2} \cup R_{3}=D^{n}$, and such that $R_{1}$ is the set of $n$-tuples that (we know) belong to $R, R_{2}$ is the set of $n$-tuples that (we know) do not belong to $R$, and $R_{3}$ is the set of $n$-tuples for which we do not know whether they belong to $R$. In the special case in which $R_{3}$ is empty, $R$ is an ordinary $n$ place relation which can be identified with $R_{1}$. A partial structure, then, is an ordered pair $\left\langle D, R_{i}\right\rangle_{i \in I}$, where $D$ is a non-empty set, and $\left(R_{i}\right)_{i \in I}$ is a family of partial relations defined over $D$. 
Now that we have the notion of a partial structure, we can consider how distinct partial structures can be related. An isomorphism between structures is a bijective mapping $f$ that preserves all of a structure's relations: $\forall x \forall y \in D\left(R x y \Longleftrightarrow R^{\prime} f(x) f(y)\right)$. A partial isomorphism between partial structures is bijective mapping that $f$ that preserves all relations of the first and second kind: $\forall x \forall y \in D\left(R_{1} x y \Longleftrightarrow R_{1}^{\prime} f(x) f(y)\right)$ and $\forall x \forall y \in$ $D\left(R_{2} x y \Longleftrightarrow R_{2}^{\prime} f(x) f(y)\right)$. In the special case in which $R_{3}$ is empty, a partial isomorphism is simply an isomorphism. A partial homomorphism can be defined analogously by dropping the requirement that the mapping is bijective. Finally, a sentence $\alpha$ is quasi-true in a partial structure $A$ just in case there is a full structure $B$ that is an 'A-normal structure' in which $\alpha$ is true in the ordinary model-theoretic (i.e., Tarskian) sense. An 'A-normal structure' is defined as one (1) which shares the domain of $A,(2)$ in which every constant of a given language is interpreted by the same object as in $A$, and (3) whose relations extend the corresponding relations in $A$ so that each relation of arity $n$, is defined for all $n$-tuples of elements of $\mathrm{D}$.

As emphasized by its defenders, this framework allows for the quasi-truth of inequivalent theories.

An important feature to note here is that a sentence and its negation can be both quasi-true. Of course, inconsistent sentences are not quasitrue in the same A-normal structure, but they can still be both quasitrue - as long as they are true in some A-normal structure. (Bueno and da Costa, 2007, 390, original emphasis)

Bueno and da Costa are concerned to defend the possibility of accepting a single scientific theory that is internally inconsistent, such a Bohr's atomic theory (cf., da Costa and French, 2003, ch.5). They propose to do this by identifying sub-theories of the inconsistent theory that are each quasi-true in the sense defined above. The case of a plurality of distinct inequivalent theories is even more clear. As Vickers observes, “...it isn't hard to see how the [partial structures] approach could be extended to cover cases of intertheory inconsistency, such as that between general relativity and quantum theory: an inconsistent theory of quantum gravity could be represented by a class of partial structures" (Vickers, 2009, 245). Because the partial structures approach allows for the quasi-truth of inequivalent (and even inconsistent) claims, one can regard more than one inequivalent theory as quasi-true. So long as the partial structures associated with each theory can be extended into corresponding full structures, then we may regard each theory as quasitrue, despite their inequivalence.

The partial structures approach offers one way of explicating the structural realist's claim that theories capture the structure of the world. On this view, when one accepts a theory, they are committing to the quasi-truth of its claims and the (partially) faithful representation of its partial structures (da Costa and French, 2003, 21). A consequence of this is that one can accept -in the partial structures sense of 'acceptance'-more than one inequivalent theory. Each of these theories will be quasi-true in the associated partial structures. 
Thus, the partial structures approach allows for RSP: genuinely inquivalent theories may be accepted in the same sense of acceptance as applies to a single theory considered in isolation.

\subsection{Real patterns}

Another way structural realists think about the theory/world relation is in terms of Dennett's real patterns. For Dennett, a real pattern is a description of some data that is more compact than the 'bit map' description. Imagine a screen made up of many pixels. The bit map description would be a specification of the state of each pixel. A more compact description might be, for instance, 'picture of Boris the cat.' The latter captures a pattern in the data as much as the former. Some patterns may be more accurate while others may be more 'noisy' but such differences don't affect their claim to reality. Could there be two real patterns in the same data that are not equivalent?

Dennett himself clearly thinks so:

...there could be two different, but equally real, patterns discernible in the noisy world. The rival theorists would not even agree on which parts of the world were pattern and which were noise, and yet nothing deeper would settle the issue. (Dennett, 1991, 49)

One of Dennett's favorite examples is Conway's Game of Life. The Life world consists of a discrete configuration of binary systems, cells that may be 'alive' or 'dead'. The state of the Life world evolves deterministically according to four simple laws. Interestingly, certain boundary conditions give rise to patterns of behavior that suggest an ontology of higher-level entities - gliders, eaters, guns, etc. - that obey their own (imperfect) higher-level laws. Moreover, it has been shown that one can build a universal computer in the Life world. One crucial step in completing this task is finding a structure to act as an infinite storage device. This is accomplished by a complex structure known as 'sliding-block memory' (Hickerson, 1990).

Now, imagine we have a Life world that instantiates the sliding block memory pattern, but with some amount of benign noise; there are some random bits here and there, but treating the system as an infinite storage device is predictively successful. There are at least two real patterns present in this Life world: (1) the pattern describing the behavior of gliders, guns and eaters; and (2) the pattern describing the behavior of an information storage device. Each pattern will be predictively successful, but they may disagree about what is part of the pattern and what is noise. For instance, a random glider would be included in pattern 1 , but not 2 . Which pattern should be taken as the correct description of the Life world? The answer, it seems, will depend on the context in which one asks the question.

This pluralism about real patterns is relevant for structural realism because several prominent structural realists have advocated understanding the theory/world relation in terms of real patterns. 
The tentative metaphysical hypothesis of this book, which is open to empirical falsification, is that there is no fundamental level, that the real patterns criterion of reality is the last word in ontology, and there is nothing more to the existence of a structure than what it takes for it to be a real pattern. (Ladyman and Ross, 2007, 178)

Ladyman and Ross claim that our best confirmed theories describe structures. Those structures are 'real' or present in the world just in case they are real patterns in (roughly) Dennett's sense. Thus, even if theories A and B ascribe different structures to the world, each could be taken to describe the structure of the world insofar as each describes a real pattern.

\subsection{General considerations}

The partial structures and real patterns approaches represent two ways in which structural realists may wish to understand the theory/world relation. Each allows for RSP; non-isomorphic structures may each be taken to capture the structure of the world in the relevant sense. Indeed, there is reason to think that any plausible form of structural realism will allow for pluralism of this kind. In light of the problem of theory change, structural realists (like traditional realists) must allow for a certain amount of 'looseness of fit' between theoretical structures and the (structure of the) world. This looseness will take different forms in different structural realist accounts, but each will admit of distinct structures that each capture the structure of the world, more or less.

Picturesquely, the structural realist sees different theories as offering different pictures of the world's structure. If we allow that they are imperfect representations, we can think of each as providing a structure that is relevantly similar to the structure of the world - if we 'squint' each of the models and the world look the same. Moreover, there is no reason to think that only one theoretical structure can stand in this relation to (the structure of) the world, thus making room for pluralism. However this crude picture is made precise, it seems likely that it will retain the features that allow for pluralism.

Traditional realism cannot make the analogous move because even 'squinting' can't render incompatible ontological commitments consistent. If, for instance, one were a traditional realist about the Game of Life case above, they would have two rival ontologies - one of gliders and another of logic gatesthat cannot be seen as approximations of one another. ${ }^{17}$ In Laudan's terms, at most such one theory can successfully refer with its central terms. More generally, inequivalent structures can each approximate the structure of the world while inequivalent ontologies cannot each approximate the ontology of

\footnotetext{
17 The realist could take each pattern to approximate the same underlying reality, namely, the bit map description. This, however, requires taking the ontologies associated with the higher-level patterns to be reducible to a more fundamental ontology. Thus, this amounts to the strategy of reinterpretation mentioned in section 2.2 and which violates Content. It also would be of no help in cases where no such underlying ontology is available, as is plausibly the case in quantum field theory ( see $\S 7$ ).
} 
the world. It is only by combining the 'looseness of fit' that is part of any plausible scientific realism with the structural realist's more limited ontological commitments that one makes space for a robust form of pluralism. ${ }^{18}$

\section{Consistency}

So far, I've argued that structural realism makes room for robust pluralism; the structural realist has the flexibility to accept a plurality of inequivalent theories with the same target. But it doesn't follow from this that there are any actual cases in which one should be pluralistic. The interesting question then becomes: When should the structural realist endorse multiple non-isomorphic theories as each (imperfectly) capturing the structure of the world? To return to the analogy with theory change, what the pluralist structural realist seeks is a synchronic analog to diachronic continuity.

There are lessons to be drawn from the diachronic case. First, strict isomorphism is too strong of a requirement. Just as one shouldn't demand that structure is preserved exactly and completely across an episode of theory change, one should not require of a synchronic plurality of theories that they be isomorphic one another. As noted above, only if one maintains that theories are perfectly accurate representations does such a demand follow, and this is something no scientific realist should endorse. Second, those aspects that are preserved are more likely to reflect genuine features of the world (Votsis, 2010). Thus, if there are common features of the plurality of structures used in the representation of a given target system, perhaps these are more likely to be features of the structure of the world. Third, theories will agree at a suitable limit with each other and other established theories. For example, special relativity and Newtonian mechanics agree at the low speed limit with each other and with other theories (e.g., those concerning the physics of materials). Such agreement at the limit functions as an institutional norm in physics, and may be required to avoid conflicts in the application of a plurality of theories. Finally, whatever else we say, some suitable notion of empirical success remains a necessary condition; any theory the realist regards as approximately true must meet the minimum requirements of success.

Thus, as a starting point, we might posit the following constraints on an acceptable plurality of theories:

Empirical success: each theory must meet the minimum conditions for empirical success.

\footnotetext{
18 The traditional realist who wishes to accommodate inconsistent ontologies will have to drop the requirement - often taken to be essential to the position - that one should commit to the existence of the ontology posited by accepted theories. Such a move will remove the central characteristic distinguishing traditional scientific realism from structural realism. One might reasonably wonder how a version of realism that isn't committed to the specific objects and properties mentioned in our best theories, but only something roughly similar to them, differs in any substantial manner from structural realism.
} 
Agreement at the limit: each theory must agree at a suitable limit with other theories in the plurality and other established theories.

Common features: common features among the plurality of theories - if there are any - are more likely to represent aspects of the world.

These conditions remain quite permissive. For instance, competing approaches to quantum mechanics might be seen as meeting the first two conditions. But, these are seen as genuine rivals by their advocates, which suggests that pluralism is inappropriate in this context. The pluralist structural realist has at least two means of response. First, she may agree that this plurality is unacceptable despite meeting the conditions; after all, these conditions are meant to be necessary, not sufficient. It seems plausible that a certain amount of good judgement on the part of scientists is required to distinguish unacceptable from acceptable pluralities. Second, the structural realist could take the revisionary position that the various interpretations are not genuine rivals. On this view, the diversity of approaches to quantum mechanics each capture structural aspects of the quantum world, even those that are genuinely inequivalent. ${ }^{19}$ If this is the right way to view the debate, the third condition suggests we should pay special attention to commonalities among the different approaches. For instance, Born's rule for calculating probabilities from quantum states appears in some form in all interpretations. ${ }^{20}$

It is worth considering a stronger condition that has been left off the list above:

Superstructure: the structures involved in any acceptable plurality must be embeddable in a single larger structure.

The partial structures approach discussed in section 4.1 is one possible motivation for such a condition. Suppose that we have two theories, associated with partial structures $S_{1}$ and $S_{2}$, respectively. Theory 1 is quasi-true in $S_{1}$ just in case there is a full structure $F_{1}$ that is an extension of $S_{1}$ and in which theory 1 is true, and similarly for theory 2, partial structure $S_{2}$ and full structure $F_{2}$. While it is no part of the approach, one might stipulate that $F_{1}=F_{2}$, that is, that there is a single full structure in which both theories are true; this could be seen as a consistency requirement. However, such a requirement limits the extent of the pluralism open to the structural realist. Theories which straightforwardly disagree about the same features of the world would be ineligible as members of an acceptable plurality. Superstructure, then, only captures the sense in which each theory is a partial representation, not an imperfect one. Robust pluralism should allow for the acceptance of theories that genuinely disagree rather than merely target different parts of the world. ${ }^{21}$

\footnotetext{
19 This seems to be the view endorsed by Chang (2012, 275).

20 This might lead to a view of quantum theory as, first and foremost, a source of probabilities (Caves et al., 2002) or logical constraints (Bub, 1974).

21 The denial of Superstructure will play an important role in the application to QFT discussed in section 7 .
} 


\section{Realism}

Given the permissiveness of pluralist structural realism, one may wonder whether it is still deserving of the title of realism. After all, I have just argued that the pluralist can endorse a plurality of theories that ascribe different structures to the same target. If the only constraints are empirical success and agreement at the limit, then does it not follow that (nearly) anything goes?

There are many ways of understanding what scientific realism demands, and to some extent the question is merely one of terminology. That said, pluralist structural realism has just as much a claim to being a form of realism as unmodified structural realism. Structural realists typically appeal to the 'no miracles' argument in defense of their realist credentials. The realist requires that some explanation be given for the astounding empirical success of our best scientific theories. The best (or only) explanation is that these theories faithfully represent the world. The structural realist cashes out 'faithful representation' in terms of capturing the structure of the world, for instance, in one of the senses discussed above. Whether structural realism satisfies the no miracles argument and the extent to which this is sufficient for realism are contentious matters.

Here I make only a more modest claim: nothing vis à vis the realism of structural realism is changed by endorsing pluralism. All that is required is that (a) theories tell us only about structure (not about traditional realist ontology) and (b) there is a certain 'looseness of fit' between the theory and the world. As I've argued above, (a) is the core of structural realism and (b) is a requirement on any plausible form of scientific realism.

Thus, pluralist structural realism is not a weakening of the view, but rather follows from its antecedent commitments. One would have to add something to standard structural realism to prevent it from allowing for robust pluralism. So, in a word, if structural realism deserves the title, so does pluralist structural realism.

\section{Application: inequivalent representations in QFT}

Finally, let's briefly consider a potential application of pluralist structural realism. ${ }^{22}$ Quantum field theory (QFT) is the framework for some of the most accurate theories in all of science. However, the ontology responsible for these successes is unclear. While in some contexts - such as scattering experimentsQFT seems to invoke a particle ontology, other contexts seem to require a field ontology. This gives rise to a challenge for the scientific realist who seeks to explain empirical success by reference to the ontology the theory describes. Moreover, unlike many other cases used to motivate pluralism, QFT is as close as we have to a fundamental theory in physics. This means that realists cannot locate a shared underlying ontology that each of the candidates - fields or particles - approximate at some higher level of description. It is incumbent

\footnotetext{
22 For a more detailed treatment, see [xxxx].
} 
on the realist to tell us what the world is like according to QFT as there is no more fundamental theory to fill in the details otherwise.

Now, there is a strong similarity between the behavior of quantum field excitations and particles in the context of QFT. Perhaps the traditional realist needn't choose between the two ontologies, but may instead regard both as approximately true descriptions of the quantum world. The difficulty with this approach is how to develop it without collapsing into a version of structural realism. It seems natural to think that what one is committing to in this case is not an ontology, but a pattern of behaviors - that is, a structure. ${ }^{23}$ One promising approach for the traditional realist is Chakravartty's suggestion to regard rival ontological proposals as specifications of different metaphysical natures the same entity could possess. However, as mentioned above ( $(2.2)$, committing to such underdescribed entities invites its own problems. At a minimum, the traditional realist who seeks to claim that particles and field excitations are sufficiently similar for the ontology of QFT to be unproblematic must provide an adequate notion of ontological similarity; otherwise, it remains unclear what is meant by one kind of ontology approximating another.

For the structural realist, by contrast, the lack of a clear ontology for QFT isn't a problem; recall that the Heisenberg and Schrödinger versions of QM also seemed to differ in their (traditional realist) ontological commitments. But, unfortunately, there are problems lurking for the structural realist as well. At least on the algebraic understanding of QFT, several inequivalent structures seem to be required. ${ }^{24}$

Candidates for the structure of a theory of $[\mathrm{QFT}]$ include abstract $\mathrm{C}^{*}$ algebras, concrete von Neumann algebras, and instances of the latter which stand to instances of the former as universal enveloping von Neumann algebras. Cast in terms of Structural Realism...: while structures of each of these types underlie some of the empirical successes of theories of $[\mathrm{QFT}]$ the collection of significant successes enjoyed by theories of [QFT] defy attribution to a single type of structure. Empirical successes mediated by the particle notion, and explanations relating the energy of quantum field states to that of their classical predecessors, require the structure of a privileged irreducible Fock space representation; such a structure stymies the physics of broken symmetry and of equilibrium at non-zero temperatures. An abstract algebraic structure broad enough to accommodate a full slate of possible dynamics for QFT

\footnotetext{
23 This may be what French and Ladyman are worried about when they complaint that "standard realism without such 'natures' is nothing more than an ersatz realism which draws on the plausibility of a structural description of theoretical objects whilst backing off from [structural realism] proper" (French and Ladyman, 2003, 36).

24 McArthur (2006) advocates pluralism in the context of the effective field theory approach to QFT. His emphasis on shared structure across different effective field theories, however, suggests a weaker pluralism than that allowed by RSP. I haven chosen to focus on Ruetsche's discussion of the algebraic approach because it provides a clear point of contact with structural realism. If the algebraic approach to QFT is misguided, then this application is a fictional one, but it nevertheless illustrates how pluralist structural realism may be of use in understanding science.
} 
in curved spacetime is too broad to sustain mean field dynamics of the sort so successful in the thermodynamic limit. The heuristically powerful insistence on Poincaré symmetry disables a full-blooded explanation of quantum coherence. And so on. (Ruetsche, 2011, 349-350)

This would mean QFT is equivocal not just about (traditional realist) ontology, but also with respect to structure. One possible structural realist response is to seek a common structure shared by the plurality. The main worry, as argued by Ruetsche, is that the obvious candidate for a shared structure the Weyl algebra ${ }^{25}$ - is too weak to support all of the applications of QFT. In particular, applications involving spontaneous symmetry breaking (SSB) and thermodynamic phenomena seem to require the use of more specific inequivalent Hilbert space representations. This suggests that the structural realist cannot rest content with the common structure shared by the inequivalent representations; doing so would leave them unable to account for important empirical successes of QFT. A central motivation for structural realism-and scientific realism in general - is to account for the empirical successes of our best scientific theories. If the structures endorsed fail to account for significant successes, then structural realism loses much of its bite. One might instead opt for an empiricist or pragmatist understanding of inequivalent representations in $\mathrm{QFT}^{26}$

French $(2012,2014)$ offers a variant of the 'common structure' approach which regards each of the inequivalent representations as possibilities unified in an underlying modal structure. The more specific structures that figure in SSB aren't a problem, claims French, because they result from a process that invokes all of the structures.

In the case of $\mathrm{QFT}, \ldots$ we cannot appeal to isomorphism, but we can identify an underlying structure. And the inequivalence is tied up with the asymmetry of the actual phenomena. In order to accommodate that, we appeal to SSB and the structural realist retains the ontological emphasis on symmetry. (French, 2014, 320)

For French, the way to resolve the challenge of inequivalent structures in QFT is to seek an "underlying structure" that they all share. This is akin to the Superstructure condition rejected above; there must be some common structure - the real structure - that underlies the success of each of the individual ones. The problem is that, as noted above, common structure alone appears too weak to account for all of the successes of QFT, so French must invoke something else (SSB), which is then claimed to be 'structural' in some

\footnotetext{
25 Roughly, the Weyl algebra is a set of operators found in every representation of the canonical commutation relations that characterize QFT. Formally, it is a $C^{*}$ algebra. For an introduction, see Ruetsche (2011, Ch.4).

26 Indeed, Ruetsche's favored 'coalescence approach' has a strong pragmatist element: "the contingent application of theories does not merely select among some preconfigured set of their contents, but genuinely alters their contents. It follows that there can be an $a$ posteriori, even a pragmatic, dimension to content specification..." Ruetsche (2011, 147, original emphasis).
} 
other sense. However, if one adopts pluralist structural realism, there needn't be such an underlying structure. While there may be important connections between the inequivalent structures deployed by physicists, they needn't be viewed in terms of a superstructure. This allows one to take the models at face value and regard each of their structures as faithfully representing the structure of the world modelled by QFT. In this way the pluralist structural realist avoids leaving hostages to fortune by rejecting the demand for a superstructure or underlying structure for any plurality of inequivalent models. As the case of QFT shows, there is some reason to be skeptical that future physics will respect such a demand.

This sentiment is nicely captured by Don Howard in his review of French and Krause (2006):

If the structural realist is committed to the view that theory affords a unique structural representation of nature, and if the algebraic point of view is the right point of view in quantum field theory, then the structural realist has a problem. My own view is that structuralism should never have committed itself to a uniqueness claim in the first place. (Howard, 2011, 231)

The robust pluralist structural realist takes up Howard's suggestion by allowing that QFT provides us with a plurality of inequivalent structures, each of which (more or less) faithfully represents the world. Note as well that the structures involved (a) agree at the limit of non-relativistic QM and (b) share an underlying structure, namely, the Weyl algebra. The former, together with the empirical success of QFT, allows that this is an acceptable plurality and the latter tells us that the Weyl algebra likely reflects a genuine feature of our world.

The diversity of candidate ontologies for QFT poses a problem for the traditional realist - that our best-confirmed physical theory resists a single ontology makes it hard to say that its success is due to getting the ontology right. However, the diversity of structures does not pose a similar problem for the structural realist, provided the arguments for adopting RSP go through; the pluralist structural realist can grant that the multiple inequivalent structures used in QFT are each successful in virtue of faithfully representing the structure of the world.

Acknowledgements Thanks to Anjan Chakravartty and the audience at the conference Scientific Knowledge Under Pluralism at the University of Pittsburgh in 2017 for providing the stimulus for this work. For their invaluable feedback on this work, thanks to Zee Perry, Alyssa Ney, Will Starr, Dean Rickles, Holger Lyre and audiences at the 2018 APA Pacific in San Diego, the 2018 Desert Philosophy Workshop in Sedona, and the symposium Structural Realism Meets Structural Representation at the University of Sydney in 2019.

\section{References}

Baker D (2009) Against field interpretations of quantum field theory. The British Journal for the Philosophy of Science 60(3):585-609 
Bub J (1974) The Interpretation of Quantum Mechanics. Springer Science \& Business Media, Dordrecht

Bueno O, da Costa NC (2007) Quasi-truth, paraconsistency, and the foundations of science. Synthese 154(3):383-399

Cartwright N (1999) The dappled world: A study of the boundaries of science. Cambridge University Press, Cambridge

Caves CM, Fuchs CA, Schack R (2002) Quantum probabilities as Bayesian probabilities. Physical review A 65(2):022305

Chakravartty A (2011) Scientific realism and ontological relativity. The Monist 94(2):157-180

Chang H (2012) Is water H2O?: Evidence, realism and pluralism. Springer Science \& Business Media

da Costa NC, French S (2003) Science and Partial Truth. Oxford University Press, Oxford

Demopoulos W, Friedman M (1985) Bertrand Russell's The analysis of matter: Its historical context and contemporary interest. Philosophy of Science $52(4): 621-639$

Dennett DC (1991) Real patterns. The Journal of Philosophy 88(1):27-51

Dupré J (1993) The disorder of things. Harvard University Press, Cambridge, MA

van Fraassen B (1980) The Scientific Image. Oxford University Press, Oxford van Fraassen B (2008a) The Empirical Stance. The Terry Lectures Series, Yale University Press

van Fraassen B (2008b) Scientific Representation. Oxford University Press, Oxford

Fraser D (2008) The fate of 'particles' in quantum field theories with interactions. Studies in History and Philosophy of Modern Physics 39(4):841-859

French S (2012) Unitary inequivalence as a problem for structural realism. Studies in History and Philosophy of Modern Physics 43(2):121 - 136

French S (2014) The Structure of the World: Metaphysics and Representation. Oxford University Press, Oxford

French S, Krause D (2006) Identity in Physics: A Historical, Philosophical, and Formal Analysis. Oxford University Press, Oxford

French S, Ladyman J (2003) Remodelling structural realism: quantum physics and the metaphysics of structure. Synthese 136-141:31-56

Halvorson H (2012) What scientific theories could not be. Philosophy of Science $79(2): 183-206$

Hickerson D (1990) Description of sliding block memory, http://www.radicaleye.com/lifepage/patterns/sbm/sbm.html

Howard D (2011) Are elementary particles individuals? A critical appreciation of Steven French and Décio Krause's Identity in physics: A historical, philosophical, and formal analysis. Metascience 20(2):225-31

Kellert SH, Longino HE, Waters CK (eds) (2006) Scientific pluralism, Minnesota studies in the philosophy of science, vol 19. University of Minnesota Press, Minneapolis 
Ladyman J (1998) What is structural realism? Studies In History and Philosophy of Science Part A 29(3):409-424

Ladyman J (2011) Structural realism versus standard scientific realism: the case of phlogiston and dephlogisticated air. Synthese 180(2):87-101

Ladyman J, Ross D (2007) Every Thing Must Go: Metaphysics Naturalized. Oxford University Press, Oxford

Laudan L (1981) A confutation of convergent realism. Philosophy of Science 48(1):19-49

Lyre H (2004) Holism and structuralism in U(1) gauge theory. Studies in History and Philosophy of Modern Physics 35(4):643 - 670

McArthur D (2006) Contra Cartwright: Structural realism, ontological pluralism and fundamentalism about laws. Synthese 151(2):233-255

Mitchell SD (2003) Biological complexity and integrative pluralism. Cambridge University Press

Morrison M (2011) One phenomenon, many models: Inconsistency and complementarity. Studies In History and Philosophy of Science Part A 42(2):342351

Newman MHA (1928) Mr. Russell's "Causal theory of perception". Mind $37(146): 137-148$

Poincaré H (1905) Science and Hypothesis, english translation edn. The Walter Scott Publishing Co., New York

Psillos S (2006) The structure, the whole structure, and nothing but the structure? Philosophy of Science 73(5):560-570

Redhead M (2001) The intelligibility of the universe. Royal Institute of Philosophy Supplement 48:73-90

Ruetsche L (2011) Interpreting Quantum Theories. Oxford University Press, Oxford

Saatsi J (2005) Reconsidering the Fresnel-Maxwell theory shift: How the realist can have her cake and EAT it too. Studies in History and Philosophy of Science Part A 36(3):509 - 538

Saunders S (1993) To what physics corresponds. In: French S, Kamminga $\mathrm{H}$ (eds) Correspondence, invariance and heuristics, Boston Studies in the Philosophy and History of Science, vol 148, Springer Netherlands, pp 295325

Vickers P (2009) Can partial structures accommodate inconsistent science? Principia 13(2):233

Vickers P (2013) Understanding Inconsistent Science. Oxford University Press, Oxford

Votsis I (2010) Structural realism: Continuity and its limits. In: Scientific structuralism, Springer, pp 105-117

Worrall J (1989) Structural realism: The best of both worlds? Dialectica 43(12):99-124 